\title{
THE MATHEMATICS OF CUMULATIVE VOTING
}

\author{
LEWIS R. MILLS*
}

The crucial tactical problem confronting each faction in a cumulative voting contest is how to cumulate their votes so as to elect the maximum number of directors possible. It was in order to provide " $a$ simplified and workable system of solving" this problem that Arthur T. Cole in 1950 published his article on the "Legal and Mathematical Aspects of Cumulative Voting." The equations presented by Cole have since become the accepted formulae for solving the mathematical problems of cumulative voting. In this article, the author demonstrates, however, that Cole's equations contain significant errors and may yield faulty results in some situations. He then sets forth a more rigorous and accurate approach to the mathematical problems involved in cumulative voting for the election of corporate directors.

A RTHUR T. COLE, JR., published his article on "Legal and
then it has been one of the standard references in discussions of

* A.B. 1951, Brown University; LL.B. 1954, Washington University; S.J.D. 1956, University of Wisconsin. Associate Professor of Law, Washington University.

12 S.G.L.Q. 225 (1950). The concept of cumulative voting in the election of corporate directors was first articulated in a provision of the lllinois state constitution adopted in 1870. Ill. Const. art. XI, \$3; see C. Williams, Cumulative Votinc for Directors 20-25 (1951); Campbell, The Origin and Growth of Cumulative Voting for Directors, Bus. LAwYer, Apr., 1955, at 3-6; Sell \& Fuge, Impact of Classified Directorates on the Constitutional Right of Cumulative Voting, 17 U. PITT. L. REv. 151, 153.56 (1956); Stephan, Cumulative Voting and Classified Boards: Some Reflections on Wolfson v. Avery, 31 NOTRE DAME LAw. 351, 351-53 (1955). Despite the novelty of the idea, it spread rapidly throughout the states, and today cumulative voting in corporate elcctions is mandatory in many states and permissive in many others. See ABA-ALl Model Bus. CoRP. Act ANN. § 31, at If 4 (1960).

Cumulative voting is designed to provide a means by which minority shareholders of a corporation can achieve representation on the board of directors commensurate with their capital interest. Under straight voting, the other common method of electing directors, each shareholder votes the number of shares he owns for each director candidate. It is obvious that, voting in this manner, a mere majority of the shareholders of a corporation can elect. all the directors, and the minority can elect none. However, under cumulative voting each shareholder has as many votes as he has shares multiplied by the number of directors to be elected, and may either concentrate all his votes on a single candidate or distribute them among several candidates as he sees fit. By utilizing the cumulative voting method and distributing their votes properly, minority shareholders of a corporation are able to attain representation on the board of directors roughly proportionate to the number of shares they own. 
cumulative voting; ${ }^{2}$ certainly, his treatment of the mathematical aspect of cumulative voting is the most ambitious in the literature. ${ }^{3}$

Unfortunately, most of the equations presented by Cole contain errors. In some instances, these errors are minor; as demonstrated below, some of his equations yield valid results except in cases in which fractional shares are permitted to vote. ${ }^{4}$ Others, however, contain more serious errors. This article demonstrates Cole's mathematical errors, and then presents a more rigorous mathematical analysis of the problem and considers some problems relating to tied elections.

\section{COLE'S ERRORS}

The first problem with which Cole deals concerns a hypothetical corporate election in which all of the outstanding shares are split between two rival factions. Let us refer to one faction as the red faction, to the other as the blue faction, and define the following symbols: ${ }^{5}$

$\mathrm{r}=$ the number of shares to be voted by the red faction.

$t=$ the total number of shares outstanding.

See generally G. WILrIAMs, supra at 6; Sell \& Fuge, supra at 151-52; Stephan, supra at 353-54.

s See, e.g., E. Aranow \& H. EInhorn, Proxy Contests for Gorporate Control 296 n.1 (1957); A. Frey, C. Morris \& J. Choper, Cases and Materiats on Corporations 308 (1966); H. Henn, Handiook of tHe LAw of Corporatrons aNd OtHER Business EnterPRISES 293 n.11 (1961); N. Lattin \& R. Jennings, Cases aNd Materials on Corporations 584 (3d ed. 1959); R. Stevens \& H. Henn, Statutes, Cases, and Materials on the Law of Corporations and Other Business Enterprises 557 n.15 (1965); ABA-ALI Model Bus. CORP. ACT ANN. $\$ 31$, at $\llbracket$ 4-5 (1960).

${ }^{3}$ Other discussions of the mathematical aspects appear in C. WILIIAMs, supra note 1, at 40-42; and Gerstenberg, The Mathematics of Cumulative Voting, $9 \mathrm{~J}$. AccoUNTANCY 117 (1910). The only equation given by Williams is the same as Cole's first equation and is subject to the same infirmities. See text accompanying note 7 infra. All of Cerstenberg's equations are given by Cole and, indeed, as Cole acknowledges, most of his mathematics are based on Gerstenberg's article. Cole, supra note 1 , at 229 n.17. Gerstenberg recognizes that the underlying mathematical relation is an inequality rather than an equation. However, he fails to develop this point. See text accompanying note 7 infra.

-See ABA-ALI MOder Bus. Corp. Act ANN. \$22 (1960), and comparable statutes described in paragraph 2 therein, for examples of statutes expressly permitting fractional shares to vote.

${ }^{\circ}$ Cole uses different symbols and designates the red faction as the minority. Cole's notation is:

$\mathrm{x}=$ the number of shares to be voted by the minority [red] faction [ $\mathrm{r}$ ]

$a=$ the total number of shares outstanding $[t]$

$\mathrm{b}=$ the total number of directors to be elected [T]

$c=$ the number of directors to be elected by the minority [red] faction $[R]$. Cole, supra note 1, at 230. 
$\mathbf{R}=$ the number of directors that the red faction can elect even if the blue faction makes optimal use of its votes.

$\mathbf{T}=$ the total number of directors to be elected.

To determine how many shares the red faction must control to elect $\mathbf{R}$ directors Cole prescribes the following equation:

$$
\mathrm{r}=\frac{\mathrm{tR}}{\mathrm{T}+\mathrm{I}}+\mathrm{l}
$$

This equation produces accurate results only if there are no fractional shares with voting rights. To illustrate the effect of fractional share voting, let us suppose that the corporation has 100 shares outstanding, a five member board of directors, and the red faction wants to elect two directors. Cole's equation becomes:

$$
r=\frac{100 \times 2}{5+1}+1=341 / 3
$$

Without mathematical explanation, Cole tells us to drop the fraction. ${ }^{6}$ Used in this manner, his equation indicates that the red faction needs to control 34 shares to elect two directors. But if there are half shares that are permitted to vote, the red faction could elect two directors if it controlled only $331 / 2$ shares. In such a case it would have 1671/2 votes; it could cast 84 votes for one candidate and $831 / 2$ votes for the other. If the blue faction split its $3321 / 2$ votes among four candidates, three of them would receive 83 votes each, and the fourth would receive $831 / 2$ votes. Consequently, both of the red faction's candidates would be elected. And, of course, if the blue faction voted for only three candidates, both of the red faction's candidates would be elected.

The basic error in Cole's approach is that he attempts to express as an equation a mathematical relation that is an inequality. ${ }^{7}$ As a result, his equation may yield inaccurate results if fractional shares vote, and it cannot be solved algebraically to produce valid equations for the other quantities, such as $\mathrm{R}$ or $\mathrm{T}$.

Cole's second equation is an application of his first to the special case in which the red faction wants to elect all the directors. Mathe-

${ }^{-}$Cole, supra note 1, at 230 \& n.19, 233, 238 n.31.

${ }^{7}$ See text accompanying note 13 infra. 
matically, this means that $R=T$. Substituting $T$ for $R$ in his first equation, Cole gives his second equation as:

$$
r=\frac{t T}{T+1}+1
$$

Similarly, Cole's third equation is an application of his first equation to the special case in which the red faction wants to elect only one director. This means that $R=1$. Substituting 1 for $R$ in his first equation, Cole gives his third equation as:

$$
r=\frac{t}{T+1}+1
$$

Both of these equations are subject to the same infirmities as the first.

Cole's fourth equation mathematically is a trivial variation of his third. He simply substracts " 1 " from the right side of his third equation to produce his fourth. He offers the fourth as a means of determining how many shares the red faction could control without being able to elect any directors:

$$
r=\frac{t}{T+1}
$$

This produces an accurate equation, but in some cases it is accurate in a very literal and somewhat surprising way. Suppose a corporation has a nine member board of directors and 100 shares outstanding, of which the red faction controls 10 shares. According to Cole's fourth equation, the red faction should be unable to elect any directors. What happens at an election? The red faction casts 90 votes for its candidate. The blue faction has 90 shares, resulting in 810 votes. If it votes for more than nine candidates, one of them will receive less than 90 votes and the red candidate will be elected. If it votes for exactly nine candidates, it can give each of them exactly 90 votes; in this event the election would be tied and no one would be elected. On these facts the blue faction prevents the red candidate's election only at a cost of not electing any blue candidates. In some jurisdictions, this could lead to liquidation of the corporation. ${ }^{8}$

${ }^{8}$ See ABA-ALI Moder Bus. Corp. Act ANN. $\$ 90$ (a) (3) (1960), and comparable statutes decribed in paragraph 2 therein, for examples of statutes permitting liquidation when shareholders are "deadlocked" and cannot elect directors. The inequality $x<\frac{t}{T+1}$ should be used in lieu of Cole's fourth equation. Values of $x$ that satisfy 
In all cases in which the voting of fractional shares is not involved, Cole's first three equations yield valid results. In one sense, then, the error in those equations is quite minor. ${ }^{9}$ But in another sense it is not. The small error in Cole's first equation may be magnified if that equation is manipulated algebraically to produce equations for other quantities. For example, if his first equation is solved for $\mathrm{R}$-the method by which he derived his fifth equation-the error becomes more serious. His fifth equation is:

$$
\mathbf{R}=\frac{(\mathrm{r}-1)(\mathrm{T}+\mathrm{I})}{\mathrm{t}}
$$

This equation, supposedly, tells the red faction how many directors it can elect if it controls $r$ shares. Suppose a corporation has 1,000 shares outstanding and has an eight member board of directors. If the red faction controls 334 shares, how many directors can it elect? Cole's fifth equation gives:

$$
\mathrm{R}=\frac{(334-1)(8+1)}{1,000}=2 \frac{997}{1,000}
$$

Because Cole tells us to drop the fraction, ${ }^{10}$ the red faction can elect only two directors-according to Cole.

But Cole is wrong. With 334 shares the red faction has 2,672 votes; it can cast 891 votes for each of two candidates and 890 votes for a third. The blue faction has 666 shares or 5,328 votes. If it spreads its votes over six candidates it can give only 888 votes to each. All three of the red faction's candidates will be elected.

Cole's sixth equation purports to apply to a slightly different factual situation. Suppose some outstanding shares are not yet committed to either the red or the blue faction. If the blue faction controls b shares, how many shares must the red faction control to elect $\mathrm{R}$ directors? Assume that the red faction can acquire the necessary amount of the uncommitted shares. Cole's sixth equation states:

$$
\mathbf{r}=\frac{\mathrm{Rb}+\mathrm{T}+1}{\mathrm{~T}-\mathrm{R}+\mathrm{I}}
$$

Cole's own example demonstrates the inaccuracy of this equation. $\mathrm{He}$ hypothesizes a corporation with 500 shares outstanding, most

this inequality are red shareholdings that do not prevent the election of all dircctors by the blue faction.

- But see statutes cited note 4 supra.

${ }^{10}$ Cole, supra note 1, at 230 n.19, 233, 238 n.31. 
of which are held by disinterested shareholders. It has a seven member board of directors. If the blue faction controls 57 shares, how many of the other shares would the red faction have to control to elect four directors? Cole says:

$$
\mathrm{r}=\frac{4 \times 57+7+1}{7-4+1}=\frac{228+8}{8-4}=\frac{236}{4}=59
$$

"According to this finding, the minority [red] faction would have to control 59 shares in order to elect four out of seven directors."11

It should be obvious (even without algebraic expressions) that if the red faction has 58 shares and the blue faction has only 57 shares, the red faction can elect the fourth director. The red faction with 58 shares would have 406 votes. It can give 101 votes to each of two candidates and 102 votes to each of two other candidates. The blue faction with 57 shares has only 399 votes. It can give 100 votes to each of three candidates and 99 to a fourth. Contrary to the result obtained from Cole's sixth equation, the red faction can elect four directors with only $\mathbf{5 8}$ shares.

Cole's seventh equation is an application of his sixth to the special case in which the red faction wants to elect all the directors. Mathematically, this means that $R=T$. Surprisingly, Cole's seventh equation cannot be obtained by substituting $T$ for $R$ in his sixth. His seventh equation is:

$$
\mathrm{r}=\mathrm{bT}+1
$$

Similarly, Cole's eighth equation is an application of his sixth to the special case in which the red faction wants to elect one director. This means $R=1$. Cole's eighth is:

$$
\mathrm{r}=\frac{\mathrm{b}}{\mathrm{T}}+\mathrm{I}
$$

Both the seventh and eighth equations can produce erroneous results if fractional shares vote. Neither can be manipulated to produce valid equations for other quantities.

Cole's ninth equation is a mathematically trivial variation of his eighth, similar to the variation on his third by his fourth. His equation gives the number of shares that the red faction can control with-

11 Id. at 235. 
out electing a director and is literally accurate in the same way that his fourth equation was. His ninth equation states:

$$
\mathrm{r}=\frac{\mathrm{b}}{\mathrm{T}}
$$

Cole's tenth equation is the same as his fifth equation except that $(r+b)$ is substituted for $t$ :

$$
R=\frac{(r-1)(T+1)}{r+b}
$$

The substitution is necessary because his tenth equation is meant to apply to cases in which not all of the outstanding shares are voted. However, the substitution does nothing to cure the basic defects in the fifth equation; consequently, the tenth equation suffers from those same defects. Cole's eleventh and twelfth equations deal with a factually different problem. ${ }^{12}$

I2 Cole's eleventh and twelfth equations attempt to deal with a more complex situation than do his first ten. They are meant to apply when there is a third faction, which we will call the yellow faction. His eleventh equation says that the yellow faction can elect one director if

$$
\mathrm{y}=\frac{\mathrm{r}}{\mathrm{R}}+\frac{1}{\mathrm{~T}}
$$

where $y$ is the number of shares controlled by the yellow faction. Id. at 237-39. In using his eleventh equation, Cole does not drop fractions; on the contrary, he treats them as whole numbers. Id. at $238 \mathrm{n} .31$. This cavalier approach to fractions makes the results-obtained valid in all cases in which fractional shares do not vote. A mathematically more precise expression (and one that holds even if fractional shares vote) is:

$$
y>\frac{\mathbf{r}}{\mathbf{R}}
$$

This expression simply states algebraically that the yellow faction can elect one director only if it has more shares per candidate than the red faction.

Cole's twelfth equation builds on his eleventh equation and deals with an improbable situation. See id. at 239-40. If the red faction knows that the blue faction controls $b$ shares, and further knows that the yellow faction will buy enough uncommitted shares to elect one director, how many shares must the red faction control to elect $\mathbf{R}$ directors? Cole's twelfth equation states:

$$
\mathrm{r}=\frac{\mathrm{bR}}{\mathrm{T}-\mathrm{R}}+1
$$

If fractions are dropped, this equation gives valid results in cases in which fractional shares do not vote. However, Cole specifically says that in using this equation, fractions are to be treated as whole numbers. Id. at $239 \mathrm{n} .33$. If this direction is followed, the equation yields a value of $x$ that is one share too high, except when $b R$ is an exact multiple of $(T-R)$.

For example, suppose the blue faction controls 50 shares and the red faction wants 


\section{A More Rigorous Approach}

We can replace Cole's spurious equations with more precise expressions. His first group of equations deal with cases in which all of the outstanding shares will be voted by one faction or the other. In such a case, the red faction has $\mathrm{r} T$ votes to distribute among $\mathbf{R}$ candidates, or $\frac{\mathrm{rT}}{\mathrm{R}}$ votes per candidate. Since all of the shares are controlled by either the red faction or the blue faction, the blue faction has $\mathrm{t}-\mathrm{r}$ shares and $(\mathrm{t}-\mathrm{r}) \mathrm{T}$ votes. Among how many candidates will the blue faction distribute its votes? If the blue faction votes for only $T-R$ candidates, there is no contest; all candidates, both red and blue, are elected. Consequently we are interested only in what would happen if the blue faction votes for more than $T-R$ candidates. But because the number of blue candidates is the denominator of the blue group's votes per candidate, the lower the number of blue candidates is, the higher their votes per candidate will be. Therefore, in order to maximize their votes per candidates in a contested election, the blue faction must vote for $(T-R+1)$ candidates. Voting for less than this number would concede the election of $\mathrm{R}$ directors by the red faction; voting for more would decrease

to elect five directors of a nine member board. How many shares does it need? Cole's equation gives

$$
\mathrm{r}=\frac{50 \times 5}{9-5}+1=621 / 2+1=631 / 2
$$

Cole would say this result means the red faction needs 64 shares. Suppose the red faction has only 63 shares; what happens at the election?

The red faction has 567 votes, it casts 113 votes for each of three candidates and 114 votes for each of two more. The blue faction has 450 votes, it casts 112 votes for each of two candidates and 113 votes for each of two more. No matter how many votes the yellow faction casts for its single candidate, none of the red candidates lose. The loser is one of the blue candidates.

The correct expression is derived as follows: Since the yellow faction is going to defeat somebody's candidate, the expression must be designed to make sure that the candidate displaced by the yellow faction is a blue candidate rather than a red one. This condition is met if the red faction's votes per candidate are greater than the blue's. Symbolically:

$$
\frac{\mathrm{IT}}{\mathrm{R}}>\frac{\mathbf{b T}}{\mathrm{T}-\mathrm{R}}
$$

This simplifies to:

$$
\mathbf{r}>\frac{\mathbf{R b}}{\mathbf{T}-\mathbf{R}}
$$

Here again Cole's equation defines a sufficient condition to achieve the desired result, but not a necessary condition. 
their votes per candidate. For this reason we can express the number of votes per blue candidate as $\frac{(t-r) T}{T-R+1}$.

For the red faction to elect its $\mathrm{R}$ candidates, its votes per candidate must be higher than the blue faction's votes per candidate. We can express this algebraically as:

$$
\frac{r T}{R}>\frac{(t-r) T}{T-R+1}
$$

This inequality can be solved for $\mathrm{r}$ in the following manner: multiplying both sides by $R(T-R+1)$ gives:

$$
\mathrm{rT}^{2}-\mathrm{rRT}+\mathrm{rT}>\mathrm{RTt}-\mathrm{rRT}
$$

Adding $\mathrm{rRT}$ to both sides and dividing both sides by $\mathrm{T}$ further simplifies it to:

$$
r(T+1)>t R
$$

Solving for $r$ yields:

$$
r>\frac{t R}{T+1}
$$

This expression means that in order to elect $\mathrm{R}$ directors, the red faction must control a number of voting shares more than (even if only fractionally more than) the quantity on the right side of the inequality. This expression is similar to but more precise than Cole's first equation. ${ }^{13}$

Moreover, this inequality can be manipulated algebraically without danger. ${ }^{14}$ If we solve it for $\mathbf{R}$ by multiplying both sides by $\frac{(\mathrm{T}+1)}{\mathrm{t}}$ we get:

$$
\mathbf{R}<\frac{\mathrm{r}(\mathrm{T}+1)}{\mathrm{t}}
$$

This expression can be used to determine how many directors the red faction can elect if it controls $r$ shares. Because $R$ is necessarily a whole number (we can have fractional shares but not fractional

\footnotetext{
${ }^{13}$ When Cole's equation is satisfied, this inequality is also satisfied. However, there are cases in which the inequality is satisfied but Cole's equation is not. In mathematical terms, the satisfaction of Cole's equation is a sufficient condition for the satisfaction of the inequality, but it is not a necessary condition.

14 An expression comparable to Cole's second equation can be obtained by substituting $T$ for $R$ in inequality (1). Similarly, an expression comparable to his third equation can be obtained by substituting 1 for $R$ in inequality ( 1 ).
} 
directors), the red faction can elect the largest whole number of directors that is smaller than the quantity on the right side of the inequality. For example, if there are 1000 shares outstanding of which the red faction controls 334 , and an eight member board of directors, the right side of the inequality is $\frac{334(8+1)}{1,000}$ or 3.006 . The largest whole number that is smaller than this quantity is, of course, three; that is the number of directors that the red faction can elect on these facts.

Inequality (1) can also be solved for $\mathrm{T}$ :

$$
\mathrm{T}>\frac{\mathrm{tR}}{\mathrm{x}}-1
$$

If the red faction has enough power to change the size of the board of directors, it can use this expression to determine how many directors there should be to permit it to elect $R$ directors with $r$ shares. Suppose the corporation has 1,000 shares outstanding. If the red faction controls 600 shares and wants to elect seven directors, what size should the board of directors be? This inequality indicates that

$$
\mathrm{T}>\frac{1000 \times 7}{600}-1
$$

The value of the right side is $10 \frac{2}{3}$; therefore, because inequality (3) requires that $\mathrm{T}$ must be larger than this amount, if the red faction sets the size of the board of directors at eleven, it can elect seven directors with 600 shares.

Cole's sixth equation failed to tell us accurately how many shares the red faction would need to elect $R$ directors, if there are uncommitted shares and the blue faction controls $\mathrm{b}$ shares. The correct mathematical approach to this problem is similar to that by which we derived inequality (1). The red faction can cast $\frac{r T}{R}$ votes for each of its $R$ candidates. If the red candidates are to be elected, this quantity must be larger than the number of votes the blue faction can cast for $T-R+1$ candidates. This condition can be stated algebraically as:

$$
\frac{\mathrm{rT}}{\mathrm{R}}>\frac{\mathrm{bT}}{\mathrm{T}-\mathrm{R}+\mathrm{I}}
$$


If we divide both sides by $\mathrm{T}$ and multiply both sides by $\mathrm{R}$, this becomes:

$$
\mathrm{r}>\frac{\mathrm{bR}}{\mathrm{T}-\mathrm{R}+\mathrm{l}}
$$

Applying this expression to Cole's hypothetical case gives

$$
\mathrm{r}>\frac{57 \times 4}{7-4+1}=57
$$

This means that the red faction must control a number of shares larger than 57 in order to elect four directors. If no fractional shares are permitted to vote, the smallest number larger than 57 is 58 , and as we saw above, 58 shares are sufficient to elect four directors. Of course, if there were fractional shares voting, fifty-seven and a fraction shares would also be sufficient.

Cole's tenth equation is meant to be used to determine how many directors the red faction can elect with $\mathrm{r}$ shares if the blue faction controls $\mathrm{b}$ shares and some of the outstanding shares do not vote. Because the non-voting shares do not affect the outcome, a correct expression for this problem can be obtained by substituting $\mathrm{r}+\mathrm{b}$ for $t$ in mequality (2):

$$
\mathrm{R}<\frac{\mathrm{r}(\mathrm{T}+\mathrm{l})}{\mathrm{r}+\mathrm{b}}
$$

This expression is a more general form of inequality (2) and can, of course, be used whether or not some outstanding shares do not vote. In applying this inequality the red faction should divide its $\mathrm{rT}$ votes among $R$ candidates in amounts as nearly equal as possible.

\section{TIED Elections}

Tied elections are troublesome, both mathematically and legally. Under cumulative voting ties can occur in two different ways. In the first type of tie, more candidates than there are directors to be elected each receive an equal number of votes, and no candidate receives more than that number of votes. For example, suppose the red faction controls 52 shares, the blue faction controls 156 shares, and the board of directors has seven members. The red faction can give 182 votes to each of two candidates; the blue faction can give 182 votes to each of six candidates; in this event, each of eight candidates for the seven-member board receives the same 
number of votes. In ties of this type none of the candidates are elected; it seems likely that the preceding board remains in office. In some jurisdictions, at least, a continued deadlock of this type is grounds for liquidation of the corporation..$^{15}$

In the second type of tie, some number of candidates less than the number of directors to be elected receive more votes than the remaining candidates. Of the remaining candidates, enough receive an equal number of votes so that the total of (i) those receiving the same number of votes, and (ii) those receiving more than that number of votes, exceeds the number of directors to be elected. For example, suppose the red faction controls 53 shares, the blue faction controls 159 shares, and the board of directors has seven members. The red faction can give 186 votes to one candidate and 185 votes to another. The blue faction can give 186 votes to each of three candidates and 185 votes to each of three more.

In ties of this second type it seems clear that some directors are elected. In the example just given, for instance, one red and three blue directors are elected. However, there appears to be no way to determine which four members of the preceding board they displace. It should follow that all members of the preceding board are displaced, leaving three vacant seats on the new board. To fill the remaining seats, perhaps there should be a run-off election among the tied candidates-an election in which all shareholders (including those who voted for the admittedly elected directors) would be permitted to vote. ${ }^{16}$ Or perhaps, in some jurisdictions, the newly elected directors should select the persons to fill the vacant seats. ${ }^{17}$

In the example just given, a run-off election should result in a deadlock-a tie of the first type. Each candidate would receive 371 votes. ${ }^{18}$ In both examples of ties given above: (i) both the red and the blue factions voted for one more candidate than inequality (5) indicated they could elect; ${ }^{19}$ and (ii) the following equation (in

\footnotetext{
${ }^{15}$ See note 8 supra.

${ }^{10}$ See State ex rel. Price v. Du Brul, 100 Ohio St. 272, 126 N.E. 87 (1919).

${ }^{17}$ See ABA-ALI MOdEr Bus. CoRP. AGT ANN. $\$ 36$ (1960), and comparable statutes described in paragraph 2 therein, for examples of statutes permitting directors in office to fill "vacancies" on the board of directors. Quaere whether such statutes are applicable to ties of the second type.

${ }^{18}$ Conceivably the applicable cumulative voting provision could be interpreted to require multiplication of the number of shares by the seats remaining to be filled rather than by the total number of directors to be elected at the election, in which case each candidate would receive 159 votes.
} 
which $R$ is the largest integer that satisfies inequality (5)) was satisfied: ${ }^{20}$

$$
\frac{\mathrm{rT}}{\mathrm{R}+1}=\frac{\mathrm{bT}}{\mathrm{T}-\mathrm{R}}
$$

Whenever these two conditions are met, a tie of either the first or second type occurs; if it is a tie of the second type it cannot be resolved by run-off elections. The possibility of ties occurring under these conditions does not impair the literal accuracy of the inequalities. It does, however, indicate the existence of an alternate strategy; by voting for $R+1$ candidates under these conditions, the red faction forces the blue faction to choose between (i) a tied election, or (ii) permitting the red faction to elect $\mathrm{R}+1$ directors.

If fractional votes are not permitted, a tie of the second type can occur in some cases even if the red faction votes for only that number of candidates that inequality (5) indicates it can elect. ${ }^{21}$ For ex-

${ }^{10}$ The designation of one faction as the red faction is, of course, wholly arbitrary. For this reason the "blue" faction cannot determine how many directors it can clect by calculating $\mathbf{R}$ from inequality (5) and then subtracting $\mathbf{R}$ from $T$. Rather, it must calculate $B$, the number of directors it can elect, from its own version of inequality (5):

$$
\mathrm{B}<\frac{\mathrm{b}(\mathrm{T}+\mathrm{l})}{\mathrm{b}+\mathrm{T}}
$$

20 This occurs whenever the right side of inequality (5) is an integer.

21 The author developed a computer program to determine, for values of $T$ up to 30 , those instances in which ties of the second type could occur even if one faction voted as indicated by inequality (5). This program was executed for the author by the Washington University Computing Facilities through National Science Foundation Grant G-22296; this assistance is gratefully acknowledged. The results for values of $T$

\begin{tabular}{|c|c|c|c|c|c|}
\hline$T$ & $\mathbf{R}$ & $\mathbf{x}$ & b & $\mathbf{R}^{\prime}$ & $\mathbf{B}^{\prime}$ \\
\hline 7 & 5 & $5 n+2$ & $3 n+1$ & 4 & 1 \\
\hline 7 & 3 & $3 n+2$ & $5 n+3$ & 2 & 1 \\
\hline 10 & 8 & $8 n+3$ & $3 n+1$ & 6 & 1 \\
\hline 10 & 3 & $3 n+2$ & $8 n+5$ & 2 & 2 \\
\hline 10 & 4 & $4 n+3$ & $7 n+5$ & 2 & 1 \\
\hline 11 & 7 & $7 n+3$ & $5 n+2$ & $\mathbf{5}$ & 2 \\
\hline 11 & 5 & $5 n+3$ & $7 n+4$ & 3 & 2 \\
\hline
\end{tabular}
through 11 are shown in the following table:

In this table $R^{\prime}$ is the number of red directors elected on the first ballot and $B^{\prime}$ is the number of blue directors elected on the first ballot. In determining the values of $r$ (the number of shares controlled by the red faction), $\mathbf{n}$ can assume the valuc of any integer, but it must have the same value in determining the corresponding value of $b$ (the number of shares controlled by the blue faction).

Some discussion of the use of this table may be helpful. Ties that affect red candidates can occur when the red faction votes as indicated by inequality (5) only if the size of the board of directors appears in the table as a possible value of $\mathrm{T}$. For example, such ties do not occur with five member boards of directors because 5 does not appear 
ample, suppose the red faction controls 53 shares, the blue faction controls 88 shares, and the board of directors has seven members. Inequality (5) indicates that the red faction can elect three directors. At an election the red faction can give 124 votes to each of two candidates and 123 votes to another. Although inequality (5) indicates that the blue faction can elect only four directors, it could give 124 votes to one candidate and 123 votes to each of four others. This would give rise to a tie of the second type in which two red and one blue director would be elected.

The possibility of such ties impairs the validity of the inequalities, although only to a limited extent. If run-off elections are used to fill the seats remaining vacant after the first ballot, the red faction will in all cases elect a total of at least $\mathrm{R}$ directors. ${ }^{22}$ When this tie-

in the table as a value of $T$. Each of the next three columns of the table imposes additional conditions on the occurrence of such ties. For example, let us see how the table is used to determine whether such a tie will occur in a specific case. Suppose the red faction controls 35 shares, the blue faction controls 57 shares, and the board of directors has seven members. Seven appears in the table as a value of $T$, so the possibility of the tie cannot be eliminated at this first stage of the analysis. Next, the value of $R$ is calculated from inequality (5). If the result is other than 5 or 3 the tie cannot occur, because these are the only values of $R$ in the table when $T$ equals 7 . In our hypothetical case, inequality (5) indicates that the red faction can elect three directors, so the possibility of a tie cannot be eliminated at the second stage of the analysis. The only value of $r$ in the table when $T$ equals 7 and $R$ equals 3 is $3 n+2$, i.e., when $r$ is divided by 3 it leaves a remainder of 2 . This condition is also met in the hypothetical case; 35 divided by 3 yields a quotient of 11 and a remainder of 2 , so the possibility of the tie cannot be eliminated at the third stage. If, however, the red faction controlled 36 or 37 shares instead of 35 , the tie could not occur; those quantities do not leave remainders of 2 when divided by 3 . The next column of the table gives a value of $b$ of $5 n+3$. At this stage, however, the value of $n$ lias been fixed as 11 , the quotient produced by dividing $r$ by 3 . This means that the tie will occur if and only if b equals $58(5 \times 11+3)$. Since in the liypothetical case $b$ is equal to 57 , we conclude that a tie does not occur if the red faction votes as indicated by inequality (5).

The tables given in note 23 infra can be used in a similar manner. In addition, all the tables can be used to construct hypothetical cases in which the specified ties occur. To do so, simply choose a line in the appropriate table, assign an arbitrary value to $n$, and calculate the resulting values of $r$ and $b$.

22 It can be demonstrated that:

$$
\frac{\mathrm{r}}{\mathrm{R}-\mathrm{R}^{\prime}}>\frac{\mathrm{b}}{\mathrm{T}-\mathrm{R}+1-\mathrm{B}^{\prime}}
$$

where $R^{\prime}$ is the number of red directors elected on the first ballot and $B^{\prime}$ is the number of blue directors elected on the first ballot. This inequality means that on the second ballot the red faction can give more votes to each of $\left(R-R^{\prime}\right)$ candidates than the blue faction can give to each of $\left(T-R+1+B^{\prime}\right)$ candidates; the red faction elects $R^{\prime}$ candidates on the first ballot and at least ( $R-R^{\prime}$ ) candidates on the second ballot, for a total of at least $R$ candidates. The proof of this inequality is too long to be given here. It requires preliminary demonstration that $r T=R \mathbf{n}+R^{\prime}, b T=$ 
breaking device is used, the inequalities predict accurately the ultimate results; a decision by the blue faction to create a tie cannot keep the red faction from electing its $\mathbf{R}$ directors.

If, however, the directors elected on the first ballot fill the remaining vacancies, the red faction may end up with less than $R$ directors. When a majority of the directors elected on the first ballot are from the blue faction, they can name all the rest of the directors. The red faction is represented only by its candidates elected on the first ballot. Because this result can occur, the inequalities are inaccurate. But because it can occur only if the board of directors has thirteen or more members, and then only in a few instances, the inequalities can be used safely in most cases. ${ }^{23}$

\section{CoNCLUSION}

Generally, Cole's equations describe conditions that are sufficient but not necessary to their predicted results. For example, if the red $(T-R+1) n+B^{\prime}$, where $n$ is any integer, and that $\frac{R^{\prime}}{R}>\frac{B^{\prime}}{T-R+1}$. From these propositions the inequality can be derived by algebraic manipulation.

${ }^{28}$ The instances in which the blue faction has a majority of the directors elected on the first ballot, for values of $T$ through 21, are:

\begin{tabular}{|c|c|c|c|c|c|}
\hline$T$ & $\mathbf{R}$ & $\mathbf{r}$ & b & $\mathbf{R}^{\prime}$ & $\underline{\mathbf{B}^{\prime}}$ \\
\hline 13 & 3 & $3 n+2$ & $11 n+7$ & 2 & 3 \\
\hline 16 & 3 & $3 n+2$ & $14 n+9$ & 2 & 4 \\
\hline 16 & 5 & $5 n+3$ & $12 n+7$ & 3 & 4 \\
\hline 17 & 5 & $5 n+2$ & $13 n+5$ & 4 & 7 \\
\hline 17 & 7 & $7 n+2$ & $11 n+3$ & 6 & \\
\hline 18 & 4 & $4 n+3$ & $15 n+11$ & 2 & 5 \\
\hline 18 & 7 & $7 n+3$ & $12 n+5$ & 5 & \\
\hline 19 & 3 & $3 n+2$ & $17 n+11$ & 2 & \\
\hline 21 & 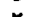 & $5 n+3$ & $17 n+10$ & 0 & \\
\hline
\end{tabular}

Note that in all of these cases $R$ is less than one-half $T$; i.e., these results can occur only when the red faction is a minority.

In other instances each faction elects the same number of directors on the first ballot. These instances are shown in the following table for values of $T$ through 21:

$\begin{array}{llllll}\frac{T}{10} & \mathbf{R} & \frac{\mathrm{T}}{\mathrm{r}} & \frac{\mathrm{b}}{\mathrm{R}^{\prime}} & \frac{\mathrm{B}^{\prime}}{2} \\ 12 & \mathbf{3} & 3 \mathrm{n}+2 & 8 \mathrm{n}+5 & 2 & 4 \\ 14 & 4 & 5 \mathrm{n}+2 & 8 \mathrm{n}+3 & 4 & 2 \\ 18 & 5 & 4 \mathrm{n}+3 & 11 \mathrm{n}+8 & 2 & 4 \\ 18 & 5 & 5 \mathrm{n}+3 & 14 \mathrm{n}+8 & 4 & 2 \\ 18 & 8 & 5 \mathrm{n}+4 & 11 \mathrm{n}+11 & 2 & 6 \\ 20 & 8 & 8 \mathrm{n}+3 & 11 \mathrm{n}+4 & 6 & 4 \\ 21 & 4 & 4 \mathrm{n}+3 & 13 \mathrm{n}+8 & 4 & 3 \\ 21 & 8 & 8 \mathrm{n}+3 & 18 \mathrm{n}+13 & 3 & 7\end{array}$

The foregoing data is from the computer study referred to in note 21 supra. 
faction has the number of shares specified by Cole's sixth equation, it can elect $\mathbf{R}$ directors. Cole's error is that $\mathbf{R}$ directors may be elected with fewer shares than his equation indicates are necessary. This article offers several inequalities that are more accurate than Cole's equations. One advantage of these inequalities is that they permit solution of problems involving the voting of fractional shares. Tied elections can occur in some cases even if one faction votes for the number of directors that the inequalities indicate it can elect. In some of those cases that faction may end up with less than the indicated number of directors. This result can occur if the directors elected on the first ballot fill the remaining vacancies. To this extent the inequalities are inaccurate. Nevertheless, for most practical problems they are sufficient. 